\title{
ESSENTIAL NORM ESTIMATES FOR HANKEL OPERATORS ON CONVEX DOMAINS IN $\mathbb{C}^{2}$
}

\author{
ŽELJKO ČUČKOVIĆ AND SÖNMEZ ŞAHUTOĞLU
}

\begin{abstract}
Let $\Omega \subset \mathbb{C}^{2}$ be a bounded convex domain with $C^{1}$-smooth boundary and $\varphi \in C^{1}(\bar{\Omega})$ such that $\varphi$ is harmonic on the nontrivial disks in the boundary. We estimate the essential norm of the Hankel operator $H_{\varphi}$ in terms of the $\bar{\partial}$ derivatives of $\varphi$ "along" the nontrivial disks in the boundary.
\end{abstract}

Let $\Omega$ be a domain in $\mathbb{C}^{n}$ for $n \geq 1$ and $b \Omega$ denote the boundary of $\Omega$. Furthermore, let $d V$ denote the volume measure on $\Omega$ and $A^{2}(\Omega)$ be the Bergman space on $\Omega$, the space of square integrable holomorphic functions on $\Omega$ with respect to $d V$. The Bergman projection $P$ is the orthogonal projection from $L^{2}(\Omega)$ onto $A^{2}(\Omega)$. For $\varphi \in L^{\infty}(\Omega)$ we define the Hankel operator $H_{\varphi}: A^{2}(\Omega) \rightarrow L^{2}(\Omega)$ by

$$
H_{\varphi} f=(I-P)(\varphi f)
$$

where $I$ denotes the identity operator on $L^{2}(\Omega)$.

In [ČŞ09] we studied compactness of Hankel operators on smooth bounded pseudoconvex domains with the symbols smooth up to the boundary. Our most complete result is attained on smooth bounded convex domains in $\mathbb{C}^{2}$. On such domains we characterize compactness of $H_{\varphi}$ in terms of the behavior of $\varphi$ on the analytic disks in $b \Omega$. Throughout this paper $\mathbb{D}$ will denote the unit open disk in $\mathbb{C}$.

Theorem ([ᄃ̌Ş09]). Let $\Omega$ be a smooth bounded convex domain in $\mathbb{C}^{2}$ and $\varphi \in C^{\infty}(\bar{\Omega})$. Then $H_{\varphi}$ is compact if and only if $\varphi \circ F$ is holomorphic for all holomorphic $F: \mathbb{D} \rightarrow b \Omega$.

In this paper we continue our study of compactness of Hankel operators and obtain estimates on their essential norms. The essential norm $\|T\|_{e}$ of a bounded linear operator $T: X \rightarrow Y$ where $X$ and $Y$ are normed linear spaces, is defined as

$$
\|T\|_{e}=\inf \{\|T-K\|: K: X \rightarrow Y \text { is a compact linear operator }\} .
$$

Date: May 4, 2022.

2010 Mathematics Subject Classification. Primary 47B35; Secondary 32W05.

Key words and phrases. Essential norm, Hankel operators, convex domains. 
That is, the essential norm of $T$ is the distance from $T$ to the subspace of compact operators.

The first estimates for the essential norms of Hankel operators were obtained by Lin and Rochberg [LR93] in 1993, for the case of the Bergman space on $\mathbb{D}$. They showed that the essential norm estimates of $H_{\varphi}$, acting on $A^{2}(\mathbb{D})$, are analogous to the estimates on the Hardy space which is a famous theorem of Adamjan, Arov and Krein [AAK71]. The Lin-Rochberg results were later generalized by Asserda [Ass00] to higher dimensions the case the domain is a strongly pseudoconvex.

As in [ČŞ09] our approach uses the connection between Hankel operators and the $\bar{\partial}$ Neumann operator. Due to this connection, we are able to consider more general domains; however, our symbols are more restricted. As a result, our estimates are of different type than Lin and Rochberg's estimates. In our case, the estimates depend on the behavior of the symbol on the analytic disks in the boundary of domains. We note that an analytic disk in the boundary of $\Omega$ is the image of a holomorphic function $F: \mathbb{D} \rightarrow b \Omega$.

Before we state our main result we define $\Gamma_{b \Omega}$, the set of all linear parametrizations of "circular" affine nontrivial analytic disks in $b \Omega$, as follows:

$$
\Gamma_{b \Omega}=\left\{F: \mathbb{D} \rightarrow b \Omega: F(\xi)=\xi z+p \text { for some } p \in b \Omega, z \in \mathbb{C}^{n} \backslash\{0\}\right\} .
$$

We note that in case there are no nontrivial affine disks in the boundary of $\Omega$, the set $\Gamma_{b \Omega}$ is empty.

In the main result below and the rest of the paper, $f_{z}$ and $f_{\bar{z}}$ denote the derivative of $f$ with respect to $z$ and $\bar{z}$ respectively.

Theorem 1. Let $\Omega$ be a $C^{1}$-smooth bounded convex domain in $\mathbb{C}^{2}, \tau_{\Omega}$ denote the diameter of $\Omega$, and $\varphi \in C^{1}(\bar{\Omega})$ such that $\varphi \circ F$ is harmonic for every holomorphic $F: \mathbb{D} \rightarrow b \Omega$. Then the Hankel operator $H_{\varphi}$ satisfies the following essential norm estimate:

$$
\sup _{F \in \Gamma_{b \Omega}}\left\{\frac{\left|F^{\prime}(0)\right|}{\sqrt{2} \tau_{\Omega}} \inf _{\xi \in \mathbb{D}}\left\{\left|(\varphi \circ F)_{\bar{\zeta}}(\xi)\right|\right\}\right\} \leq\left\|H_{\varphi}\right\|_{e} \leq \sup _{F \in \Gamma_{b \Omega}}\left\{\frac{\sqrt{e} \tau_{\Omega}}{\left|F^{\prime}(0)\right|} \sup _{\xi \in \mathbb{D}}\left\{\left|(\varphi \circ F)_{\bar{\zeta}}(\xi)\right|\right\}\right\} .
$$

Remark 1. Both estimates in the theorem above are defined to be zero in case $\Gamma_{b \Omega}=\varnothing$. That is, in case there are no nontrivial analytic disks in $b \Omega$ we get $\left\|H_{\varphi}\right\|_{e}=0$. This is in accordance with the fact that, in this case, $H_{\varphi}$ is compact. 
Remark 2. $F^{\prime}(0)$ measures the size of the disk $F(\mathbb{D}) \subset b \Omega$. So it is interesting that the essential norm depends on the "bar" derivatives of $\varphi$ on the disks in the boundary as well as the size of these disks.

In case of the bidisk we get a better estimate for the lower bound as in the following theorem.

Theorem 2. Let $\varphi \in C^{1}\left(\overline{\mathbb{D}^{2}}\right)$ such that the functions $z \rightarrow \varphi\left(z, e^{i \theta}\right)$ and $w \rightarrow \varphi\left(e^{i \theta}, w\right)$ are harmonic on $\mathbb{D}$ for all $\theta \in[0,2 \pi]$. Then the Hankel operator $H_{\varphi}$ satisfies the following essential norm estimate:

$$
\left\|H_{\varphi}\right\|_{e} \geq \sup _{F \in \Gamma_{b \mathbb{D}^{2}}}\left\{\frac{\left|F^{\prime}(0)\right|}{\sqrt{2}} \inf _{\xi \in \mathbb{D}}\left\{\left|(\varphi \circ F)_{\bar{\xi}}(\xi)\right|\right\}\right\}
$$

Remark 3. The diameter of the bidisk $\tau_{\mathbb{D}^{2}}=2 \sqrt{2}$ is the distance between $(-1,-1)$ and $(1,1)$. Hence $\sqrt{2} \tau_{\mathbb{D}^{2}}=4>\sqrt{2}$. Thus the lower bound in Theorem 2 is better than the one in Theorem 1 .

\section{PROOFS OF THEOREM 1 AND 2}

Lemma 1. Let $\gamma \in C_{0}^{1}(U)$ where $U \subset \mathbb{D}$ is a domain. Then $\left\|\gamma_{\bar{\xi}}\right\|=\left\|\gamma_{\bar{\zeta}}\right\|$.

Proof. Since $\gamma$ is compactly supported in $U$ there are no boundary terms in the following integration by parts formula.

$$
\left\|\gamma_{\xi}\right\|^{2}=\int_{U} \gamma_{\bar{\zeta}}(\xi) \bar{\gamma}_{\bar{\xi}}(\xi) d V(\xi)=\int_{U} \gamma(\xi) \bar{\gamma}_{\bar{\xi} \xi}(\xi) d V(\xi)=\int_{U} \gamma_{\bar{\zeta}}(\xi) \bar{\gamma}_{\xi}(\xi) d V(\xi)=\left\|\gamma_{\bar{\xi}}\right\|^{2}
$$

Therefore, $\left\|\gamma_{\bar{\xi}}\right\|=\left\|\gamma_{\bar{\xi}}\right\|$.

We note that a unitary affine mapping $F$ on $\mathbb{C}^{n}$ is of the form $F(z)=A z+p$ where $A$ is a $n \times n$ unitary matrix and $p \in \mathbb{C}^{n}$.

Lemma 2. Let $V$ be a bounded domain in $\mathbb{C}^{n}, F$ be a unitary affine mapping, and $\phi \in L^{\infty}(V)$. Then $\left\|H_{\phi}\right\|_{e}=\left\|H_{\phi \circ F}\right\|_{e}$ where $H_{\phi \circ F}$ is the Hankel operator (with symbol $\phi \circ F$ ) on $A^{2}\left(F^{-1}(V)\right)$.

Proof. Let $U=F^{-1}(V)$ and the pull-back $F^{*}: A^{2}(V) \rightarrow A^{2}(U)$ be defined as $F^{*}(f)=$ $f \circ F$ for $f \in A^{2}(V)$. Then one can check that $F^{*}$ is an isometry. Furthermore, the Bergman kernel transformation formula of Bell (see, [JP93, Proposition 6.1.7]) $P^{V}=\left(F^{-1}\right)^{*} P^{U} F^{*}$ where $P^{U}, P^{V}$ are the Bergman projections on $U$ and $V$, respectively. Then for $f \in A^{2}(V)$ 
we have

$$
\begin{aligned}
\left(F^{-1}\right)^{*} H_{\phi \circ F} F^{*}(f) & =\left(F^{-1}\right)^{*} H_{\phi \circ F}(f \circ F) \\
& =\left(F^{-1}\right)^{*}\left(\phi(F) f(F)-P^{U}(\phi(F) f(F))\right) \\
& =\phi f-\left(F^{-1}\right)^{*} P^{U} F^{*}(\phi f) \\
& =\phi f-P^{V}(\phi f) \\
& =H_{\phi}(f)
\end{aligned}
$$

Also $T^{V}: A^{2}(V) \rightarrow L^{2}(V)$ is a compact linear operator if and only if $T^{U}: A^{2}(U) \rightarrow L^{2}(U)$ is compact where $T^{V}=\left(F^{-1}\right)^{*} T^{U} F^{*}$. Furthermore,

$$
\left\|H_{\phi}-T^{V}\right\|=\left\|\left(F^{-1}\right)^{*} H_{\phi \circ F} F^{*}-\left(F^{-1}\right)^{*} T^{U} F^{*}\right\|=\left\|H_{\phi \circ F}-T^{U}\right\| .
$$

One can check that, the equality above implies that $\left\|H_{\phi}\right\|_{e}=\left\|H_{\phi \circ F}\right\|_{e}$.

We will use the $\bar{\partial}$-Neumann problem to obtain the upper bound in Theorem 1 . The $\bar{\partial}$-Neumann operator, denoted by $N$, is defined as the solution operator for the complex Laplacian $\overline{\partial \partial}^{*}+\bar{\partial}^{*} \bar{\partial}$ on square integrable $(0,1)$-forms on $\Omega$, denoted by $L_{(0,1)}^{2}(\Omega)$. We refer the reader to the books [CS01, Str10] and references there in, for more information about the $\bar{\partial}$-Neumann problem. In the following theorem we list the properties we need about $N$ (see [CS01, Theorem 4.4.1]).

Theorem. Let $\Omega$ be a bounded pseudoconvex domain in $\mathbb{C}^{n}$ for $n \geq 2$. There exists a bounded self-adjoint operator $N: L_{(0,1)}^{2}(\Omega) \rightarrow L_{(0,1)}^{2}(\Omega)$ such that

i. $\left(\bar{\partial}^{*} \bar{\partial}+\overline{\partial \partial}^{*}\right) N=$ I on $L_{(0,1)}^{2}(\Omega)$,

ii. $\bar{\partial}^{*} N$ is the solution operator to $\bar{\partial} u=v$ that produces solutions orthogonal to $A^{2}(\Omega)$,

iii. the Bergman projection P satisfies the following equality

$$
P=I-\bar{\partial}^{*} N \bar{\partial}
$$

where $I$ is the identity mapping,

iv. the operators $\bar{\partial} N, \bar{\partial}^{*} N, \overline{\partial \partial}^{*} N$, and $\bar{\partial}^{*} \bar{\partial} N$ are bounded and

$$
\|N\| \leq e \tau_{\Omega}^{2}, \quad\|\bar{\partial} N\| \leq \sqrt{e} \tau_{\Omega}, \quad\left\|\bar{\partial}^{*} N\right\| \leq \sqrt{e} \tau_{\Omega}
$$

where $\tau_{\Omega}$ is the diameter of $\Omega$. 
We note that ii. and iii. in the theorem above imply that $\bar{\partial} H_{\varphi} f=f \bar{\partial} \varphi$ for $\varphi \in C^{1}(\bar{\Omega})$ and $f \in A^{2}(\Omega)$.

Remark 4. Before we start the proof of Theorem 1 we note that even though [ČŞ09, Corollary 2] is stated for $\varphi \in C^{\infty}(\bar{\Omega})$, observation of the proof reveals that it is enough to have $C^{1}$-smooth domain $\Omega$ and $\varphi \in C^{1}(\bar{\Omega})$.

Proof of Theorem 1 First we will prove the lower bound. Since $\Omega$ is a $C^{1}$-smooth bounded convex domain in $\mathbb{C}^{2}$, [ČŞ09. Corollary 2] implies that $H_{\varphi}$ is compact if and only if $\varphi \circ F$ is holomorphic for any holomorphic $F: \mathbb{D} \rightarrow b \Omega$. Thus, in order to find the essential norm estimate, without loss of generality, we assume that there exists holomorphic $F: \mathbb{D} \rightarrow b \Omega$ such that $(\varphi \circ F)_{\bar{\zeta}} \not \equiv 0$. Since $\varphi$ is $C^{1}$-smooth, this means that $\varphi_{\bar{z}}(F) \neq 0$ on some open set. But the domain $\Omega$ is convex which implies that the disk $F(\mathbb{D})$ is an affine disk (see [FS98, Ц̌SS09]). Using Lemma 2 we can thus assume that there exists $\tau_{0} \in\left(0, \tau_{\Omega}\right)$ such that

i. $\varphi_{\bar{z}}(z, 0) \neq 0$ for all $|z| \leq \tau_{0}$,

ii. $\left\{(z, w) \in \mathbb{C}^{2}:|z| \leq \tau_{0}, w=0\right\} \subset b \Omega$.

Since $\Omega$ is bounded we can also deduce that

iii. $\Omega \subset\left\{z \in \mathbb{C}:|z|<\tau_{\Omega}\right\} \times\left\{w \in \mathbb{C}:|w|<\tau_{\Omega}, \operatorname{Re}(w)>0\right\}$.

With this setup, we can now put a wedge $W$ in $\Omega$ perpendicular to $D=\left\{z \in \mathbb{C}:|z|<\tau_{0}\right\}$. Furthermore, $W$ can be chosen as close to flat as we want if we are willing to choose its radius very small. That is, for any $\varepsilon_{1}>0$ there exists $r_{0}>0$ so that $D \times W \subset \Omega$ where

$$
W=\left\{r e^{i \theta} \in \mathbb{C}: 0 \leq r<r_{0},|\theta|<\frac{\pi-\varepsilon_{1}}{2}\right\} \text {. }
$$

Let us choose

$$
\chi(z)=\frac{2}{\pi \tau_{0}^{2}}\left(1-\frac{|z|^{2}}{\tau_{0}^{2}}\right) \text { for } z \in \bar{D} .
$$

Then $\chi \in C^{\infty}(\bar{D}), \chi \geq 0, \chi(z)=0$ for $|z|=\tau_{0}$. Then we have

$$
\int_{D} \chi(z) d V(z)=\frac{2}{\pi \tau_{0}^{2}} 2 \pi \int_{0}^{\tau_{0}}\left(\rho-\frac{\rho^{3}}{\tau_{0}^{2}}\right) d \rho=\frac{4}{\tau_{0}^{2}}\left(\frac{\tau_{0}^{2}}{2}-\frac{\tau_{0}^{4}}{4 \tau_{0}^{2}}\right)=1,
$$

and

$$
\left\|\chi_{z}\right\|^{2}=\frac{4}{\pi^{2} \tau_{0}^{4}} \int_{D} \frac{|z|^{2}}{\tau_{0}^{4}} d V(z)=\frac{4}{\pi^{2} \tau_{0}^{8}} 2 \pi \int_{0}^{\tau_{0}} \rho^{3} d \rho=\frac{8}{\pi \tau_{0}^{8}} \frac{\tau_{0}^{4}}{4}=\frac{2}{\pi \tau_{0}^{4}}
$$


Hence

$$
\frac{\int_{D} \chi(z) d V(z)}{\left\|\chi_{z}\right\|}=\tau_{0}^{2} \sqrt{\frac{\pi}{2}}=\frac{V(D)}{\sqrt{2 \pi}}
$$

Let us first restrict $\varphi$ onto $D$ and extend the restriction as a $C^{1}$-smooth function $\phi_{1}$ defined on $\mathbb{C} \times\{0\}$. Finally, we extend the function $\phi_{1}$ trivially as a $C^{1}$-smooth function $\varphi_{1}$ on $\mathbb{C}^{2}$. That is, $\varphi_{1}(z, w)=\varphi(z, 0)$. Let us define $\varphi_{2}=\varphi-\varphi_{1}$ and

$$
\gamma(z)=\frac{\chi(z)}{\varphi_{1 \bar{z}}(z, 0)} \text { for } z \in \bar{D}
$$

where $\varphi_{1 \bar{z}}$ denotes $\frac{\partial \varphi_{1}}{\partial \bar{z}}$. We will continue to use this notation below when appropriate.

We note that, in the rest of the proof $\|$.$\| and \|.\|_{U}$ denote the $L^{2}$ norm on $\Omega$ and on open set $U$, respectively.

Let us define $\alpha_{j}=1-2^{-2 j-1}$ and

$$
f_{j}(z, w)=\frac{1}{2^{j} w^{\alpha_{j}}} \text { for }(z, w) \in \Omega .
$$

Using polar coordinates one can show that

$$
\left\|f_{j}\right\|_{W}=\sqrt{\pi-\varepsilon_{1}} r_{0}^{1-\alpha_{j}} \text { and }\left\|f_{j}\right\| \leq \pi \tau_{\Omega}^{2-\alpha_{j}} .
$$

We will use the following equality in the second equality in (2) below.

$$
\frac{\partial H_{\varphi_{1}} f_{j}}{\partial \bar{z}} d \bar{z}+\frac{\partial H_{\varphi_{1}} f_{j}}{\partial \bar{w}} d \bar{w}=\bar{\partial} H_{\varphi_{1}} f_{j}=\bar{\partial}\left(\varphi_{1} f_{j}-P\left(\varphi_{1} f_{j}\right)\right)=f_{j} \bar{\partial} \varphi_{1}=f_{j} \frac{\partial \varphi_{1}}{\partial \bar{z}} d \bar{z} .
$$

Then, for $w \in W$ we have

$$
\begin{aligned}
\frac{1}{2^{j} w^{\alpha_{j}}} \int_{D} \chi(z) d V(z) & =\int_{D} f_{j}(z, w) \frac{\partial \varphi_{1}}{\partial \bar{z}}(z, w) \gamma(z) d V(z) \\
& =\int_{D} \frac{\partial H_{\varphi_{1}} f_{j}}{\partial \bar{z}}(z, w) \gamma(z) d V(z) \\
& =-\int_{D} H_{\varphi_{1}} f_{j}(z, w) \gamma_{\bar{z}}(z) d V(z) .
\end{aligned}
$$

We note that in the last equality above we used integration by parts and the fact that $\gamma(z)=0$ for $|z|=\tau_{0}$.

Now we take the absolute values of both sides of (2) and then apply the CauchySchwarz inequality to the right hand side to get

$$
\left|f_{j}(0, w)\right| \int_{D} \chi(z) d V(z) \leq\left\|H_{\varphi_{1}} f_{j}\right\|_{D}\left\|\gamma_{\bar{z}}\right\|_{D} .
$$


After integrating over the wedge $W$ and dividing by $\left\|\gamma_{\bar{z}}\right\|_{D}$ we get

$$
\left\|f_{j}(0, .)\right\|_{W} \frac{\int_{D} \chi(z) d V(z)}{\left\|\gamma_{\bar{z}}\right\|_{D}} \leq\left\|H_{\varphi_{1}} f_{j}\right\|_{D \times W} \leq\left\|H_{\varphi_{1}} f_{j}\right\|
$$

We remind the reader that $\varphi_{1 \bar{z}}$ and $\varphi_{1 z \bar{z}}$ below will denote $\frac{\partial \varphi_{1}}{\partial \bar{z}}$ and $\frac{\partial^{2} \varphi_{1}}{\partial z \partial \bar{z}}$ respectively. Since we assumed that $\varphi$ is harmonic on $D$, Lemma 1 implies that

$$
\left\|\gamma_{\bar{z}}\right\|_{D}=\left\|\gamma_{z}\right\|_{D}=\left\|\frac{\chi_{z}}{\varphi_{1 \bar{z}}}-\chi \frac{\varphi_{1 z \bar{z}}}{\left(\varphi_{1 \bar{z}}\right)^{2}}\right\|_{D}=\left\|\frac{\chi_{z}}{\varphi_{1 \bar{z}}}\right\|_{D} \leq \frac{\left\|\chi_{z}\right\|_{D}}{\inf _{D}\left|\varphi_{1 \bar{z}}\right|}
$$

Then

$$
\left\|H_{\varphi_{1}} f_{j}\right\| \geq \frac{\int_{D} \chi(z) d V(z)}{\left\|\gamma_{\bar{z}}\right\|_{D}}\left\|f_{j}(0, .)\right\|_{W} \geq \frac{\int_{D} \chi(z) d V(z)}{\left\|\chi_{z}\right\|_{D}}\left(\inf _{D}\left|\varphi_{1 \bar{z}}\right|\right)\left\|f_{j}(0, .)\right\|_{W} .
$$

Therefore, inequality (3) and the fact that $\left\|f_{j}(0, .)\right\|_{W}=\sqrt{\pi-\varepsilon_{1}} r_{0}^{1-\alpha_{j}}$ imply that

$$
\left\|H_{\varphi_{1}} f_{j}\right\| \geq r_{0}^{1-\alpha_{j}} \sqrt{\frac{\pi-\varepsilon_{1}}{2 \pi}} V(D)\left(\inf _{D}\left|\varphi_{1 \bar{z}}\right|\right) \text {. }
$$

Now we turn to $\varphi_{2}$. Since $\varphi_{2}(z, 0)=0$, for every $\varepsilon>0$ there exists $\delta>0$ and $j_{\varepsilon}$ so that

i. $\left|\varphi_{2}(z, w)\right|<\varepsilon$ for $(z, w) \in \bar{\Omega}$ and $|w| \leq \delta$,

ii. $\left|f_{j}(z, w)\right|<\varepsilon$ for $(z, w) \in \bar{\Omega},|w| \geq \delta$ and $j \geq j_{\varepsilon}$.

Let us denote $\Omega_{1, \delta}=\{(z, w) \in \Omega:|w|<\delta\}$ and $\Omega_{2, \delta}=\{(z, w) \in \Omega:|w|>\delta\}$. Then for $j \geq j_{\varepsilon}$ we have

$$
\begin{aligned}
\left\|H_{\varphi_{2}} f_{j}\right\| & \leq\left\|\varphi_{2} f_{j}\right\| \\
& =\left\|\varphi_{2} f_{j}\right\|_{\Omega_{1, \delta}}+\left\|\varphi_{2} f_{j}\right\|_{\Omega_{2, \delta}} \\
& \leq \varepsilon\left(\left\|f_{j}\right\|+\left\|\varphi_{2}\right\|\right) \\
& \leq \varepsilon\left(\pi \tau_{\Omega}^{2-\alpha_{j}}+\left\|\varphi_{2}\right\|\right) .
\end{aligned}
$$

Then, $\lim \sup _{j \rightarrow \infty}\left\|H_{\varphi_{2}} f_{j}\right\| \leq \varepsilon\left(\pi \tau_{\Omega}+\left\|\varphi_{2}\right\|\right)$. Since $\varepsilon$ is arbitrary, we get

$$
\lim _{j \rightarrow \infty}\left\|H_{\varphi_{2}} f_{j}\right\|=0 .
$$

By the definition of essential norms for Hankel operators, for any $\varepsilon_{2}>0$ there exists a compact operator $K_{\varepsilon_{2}}: A^{2}(\Omega) \rightarrow L^{2}(\Omega)$ such that

$$
\left\|H_{\varphi}\right\|_{e} \geq\left\|H_{\varphi}-K_{\varepsilon_{2}}\right\|-\varepsilon_{2} .
$$


Then

$$
\begin{aligned}
\left\|H_{\varphi}\right\|_{e} & \geq \limsup _{j \rightarrow \infty} \frac{\left\|H_{\varphi} f_{j}-K_{\varepsilon_{2}} f_{j}\right\|}{\pi \tau_{\Omega}^{2-\alpha_{j}}}-\varepsilon_{2} \\
& \geq \limsup _{j \rightarrow \infty} \frac{\left\|H_{\varphi_{1}} f_{j}\right\|-\left\|H_{\varphi_{2}} f_{j}\right\|-\left\|K_{\varepsilon_{2}} f_{j}\right\|}{\pi \tau_{\Omega}^{2-\alpha_{j}}}-\varepsilon_{2} \\
& =\limsup _{j \rightarrow \infty} \frac{\left\|H_{\varphi_{1}} f_{j}\right\|}{\pi \tau_{\Omega}^{2-\alpha_{j}}-\varepsilon_{2} .}
\end{aligned}
$$

In the last equality we used (5), compactness of $K_{\varepsilon_{2}}$, and the fact that $f_{j} \rightarrow 0$ weakly. Therefore, combining (4) and (6) together with the fact that the constants $\varepsilon_{1}, \varepsilon_{2}>0$ are arbitrary we get

$$
\left\|H_{\varphi}\right\|_{e} \geq \frac{1}{\sqrt{2} \pi \tau_{\Omega}} \sup _{D \subset b \Omega}\left\{V(D) \inf _{\xi \in D}\left\{\left|\varphi_{\bar{z}}(\xi)\right|\right\}\right\} .
$$

We note that there is a one-to-one correspondence between the (affine) disks in $b \Omega$ and $F \in \Gamma_{b \Omega}$. Since we need $F: \mathbb{D} \rightarrow D$ to be a surjection we must have $F(\xi)=\left(\tau_{0} \xi, 0\right)$. Then one can show that

$$
V(D) \inf _{\xi \in D}\left\{\left|\varphi_{\bar{z}}(\xi)\right|\right\}=\pi\left|F^{\prime}(0)\right| \inf _{\xi \in \mathbb{D}}\left\{\left|(\varphi \circ F)_{\bar{\zeta}}(\xi)\right|\right\} .
$$

Therefore, we have

$$
\left\|H_{\varphi}\right\|_{e} \geq \sup _{F \in \Gamma_{b \Omega}}\left\{\frac{\left|F^{\prime}(0)\right|}{\sqrt{2} \tau_{\Omega}} \inf _{\xi \in \mathbb{D}}\left\{\left|(\varphi \circ F)_{\bar{\zeta}}(\xi)\right|\right\}\right\} .
$$

Now we turn to the upper estimate. Let $\rho$ be a defining function for $\Omega$. That is, $\rho$ is a $C^{1}$-smooth function in a neighborhood of $\bar{\Omega}$ such that $\rho<0$ on $\Omega, \rho>0$ on $\mathbb{C}^{2} \backslash \bar{\Omega}$, and $|\nabla \rho| \neq 0$ on $b \Omega$. Then we define the complex tangential and complex normal vector fields as

$$
\begin{aligned}
& L_{1}=\frac{2 \sqrt{2}}{\|\nabla \rho\|}\left(\frac{\partial \rho}{\partial w} \frac{\partial}{\partial z}-\frac{\partial \rho}{\partial z} \frac{\partial}{\partial w}\right) \\
& L_{2}=\frac{2 \sqrt{2}}{\|\nabla \rho\|}\left(\frac{\partial \rho}{\partial \bar{z}} \frac{\partial}{\partial z}+\frac{\partial \rho}{\partial \bar{w}} \frac{\partial}{\partial w}\right) .
\end{aligned}
$$

One can check that $\left\{L_{1}, L_{2}\right\}$ form a continuous orthonormal basis for the space of $(1,0)$ type vector fields on a neighborhood on $b \Omega$. Let $\omega_{1}$ and $\omega_{2}$ be the differential forms of 
type $(1,0)$ that are the dual to $L_{1}$ and $L_{2}$, respectively. That is,

$$
\begin{aligned}
& \omega_{1}=\frac{\sqrt{2}}{\|\nabla \rho\|}\left(\frac{\partial \rho}{\partial \bar{w}} d z-\frac{\partial \rho}{\partial \bar{z}} d w\right) \\
& \omega_{2}=\frac{\sqrt{2}}{\|\nabla \rho\|}\left(\frac{\partial \rho}{\partial z} d z+\frac{\partial \rho}{\partial w} d w\right) .
\end{aligned}
$$

One can check that $\left\|\omega_{1}\right\|=\left\|\omega_{2}\right\|=1$ and $\bar{\partial} f=\bar{L}_{1}(f) \bar{\omega}_{1}+\bar{L}_{2}(f) \bar{\omega}_{2}$ for any $f \in C^{1}(\bar{\Omega})$ (see special boundary charts in [Str10, p. 12]).

Using the method in the first part of the proof of Theorem 3 in [ČSO9, p. 3739-3740] ( $\widetilde{\beta}$ and $\widehat{\beta}$ in [ČŞ09] correspond to $\varphi_{3}$ and $\varphi_{4}$ below, respectively) we define $\varphi_{3}, \varphi_{4} \in C^{1}(\bar{\Omega})$ such that

i. $\varphi=\varphi_{3}+\varphi_{4}$

ii. $\varphi_{3}=\varphi$ and $\bar{L}_{2}\left(\varphi_{3}\right)=0$ on $b \Omega$,

iii. $\varphi_{4}=0$ on $b \Omega$.

We note that $\varphi_{4}$ is a uniform limit of compactly supported smooth functions on $\Omega$. This fact together with Montel's Theorem imply that $H_{\varphi_{4}}$ is a limit of compact operators in the operator norm. Hence $H_{\varphi_{4}}$ is compact and $\left\|H_{\varphi}\right\|_{e}=\left\|H_{\varphi_{3}}\right\|_{e}$.

Let

$$
\Pi=\overline{\bigcup_{F \in \Gamma_{b \Omega}} F(\mathbb{D})}
$$

and $\chi_{\varepsilon} \in C^{\infty}(\bar{\Omega})$ such that $0 \leq \chi_{\varepsilon} \leq 1, \chi_{\varepsilon}=1$ on $\Pi_{\varepsilon}=\{z \in \bar{\Omega}: d(z, \Pi) \leq \varepsilon\}$, and $\chi_{\varepsilon}=0$ on $\bar{\Omega} \backslash \Pi_{2 \varepsilon}$. Then for $f \in A^{2}(\Omega)$ we have

$$
H_{\varphi_{3}}=\bar{\partial}^{*} N M_{\bar{\partial} \varphi_{3}}=\bar{\partial}^{*} N M_{\chi_{\varepsilon} \bar{\partial} \varphi_{3}}+\bar{\partial}^{*} N M_{\left(1-\chi_{\varepsilon}\right)} \bar{\partial} \varphi_{3}
$$

where $M_{h}$ denotes the multiplication by $h$. First we will show that $\bar{\partial}^{*} N M_{\left(1-\chi_{\varepsilon}\right) \bar{\partial} \varphi_{3}}$ is compact on $A^{2}(\Omega)$. Let $f \in A^{2}(\Omega)$.

$$
\begin{aligned}
\left\|\bar{\partial}^{*} N f\left(1-\chi_{\varepsilon}\right) \bar{\partial} \varphi_{3}\right\|^{2} & =\left\langle\bar{\partial}^{*} N f\left(1-\chi_{\varepsilon}\right) \bar{\partial} \varphi_{3}, \bar{\partial}^{*} N f\left(1-\chi_{\varepsilon}\right) \bar{\partial} \varphi_{3}\right\rangle \\
& =\left\langle f \bar{\partial} \varphi_{3},\left(1-\chi_{\varepsilon}\right) N \overline{\partial \partial}^{*} N f\left(1-\chi_{\varepsilon}\right) \bar{\partial} \varphi_{3}\right\rangle \\
& \lesssim\|f\|\left\|\left(1-\chi_{\varepsilon}\right) N \overline{\partial \partial}^{*} N f\left(1-\chi_{\varepsilon}\right) \bar{\partial} \varphi_{3}\right\|
\end{aligned}
$$

Now we will use the fact that $\left(1-\chi_{\varepsilon}\right) N$ is compact. This is essentially done on pages 3740-3741 in the proof of Theorem 3 in [Čş09]. The idea is to use compactness of $\bar{\partial}$ Neumann operator locally to get the following compactness estimate: for every $\varepsilon_{1}>0$ 
there exists a compact operator $K_{\varepsilon_{1}}$ on $L_{(0,1)}^{2}(\Omega)$ so that

$$
\left\|\left(1-\chi_{\varepsilon}\right) N h\right\| \leq \varepsilon\|h\|+\left\|K_{\varepsilon_{1}} h .\right\|
$$

Then using the fact that $\overline{\partial \partial}^{*} N$ is a bounded operator in the second inequality below, we get

$$
\begin{aligned}
\left\|\left(1-\chi_{\varepsilon}\right) N \overline{\partial \partial}^{*} N f\left(1-\chi_{\varepsilon}\right) \bar{\partial} \varphi_{3}\right\| & \leq \varepsilon_{1}\left\|\overline{\partial \partial}^{*} N f\left(1-\chi_{\varepsilon}\right) \bar{\partial} \varphi_{3}\right\|+\left\|K_{\varepsilon_{1}} \bar{\partial}^{*} N f\left(1-\chi_{\varepsilon}\right) \bar{\partial} \varphi_{3}\right\| \\
& \lesssim \varepsilon_{1}\|f\|+\left\|\widetilde{K}_{\varepsilon_{1}} f\right\|_{(0,1)}
\end{aligned}
$$

where $\widetilde{K}_{\varepsilon_{1}}=K_{\varepsilon_{1}} \overline{\partial \partial}^{*} N M_{\left(1-\chi_{\varepsilon}\right) \bar{\partial} \varphi_{3}}$ is a compact operator. Therefore, $\bar{\partial}^{*} N M_{\left(1-\chi_{\varepsilon}\right) \bar{\partial} \varphi_{3}}$ satisfies a compactness estimate and hence it is compact. Then

$$
\bar{\partial} \varphi_{3}=\bar{L}_{1}\left(\varphi_{3}\right) \bar{\omega}_{1}+\bar{L}_{2}\left(\varphi_{3}\right) \bar{\omega}_{2} .
$$

Using the facts that $\bar{L}_{2} \varphi_{3}=0$ and $\varphi=\varphi_{3}$ on $b \bar{\Omega}$ we get

$$
\left|\bar{\partial} \varphi_{3}\right|=\left|\bar{L}_{1}\left(\varphi_{3}\right)\right|=\left|\bar{L}_{1}(\varphi)\right| \text { on } b \Omega \text {. }
$$

Therefore, we have

$$
\left\|\bar{\partial}^{*} N f \chi_{\varepsilon} \bar{\partial} \varphi_{3}\right\| \leq\left\|\bar{\partial}^{*} N\right\|\left\|f \chi_{\varepsilon} \bar{\partial} \varphi_{3}\right\| \leq\left\|\bar{\partial}^{*} N\right\| \sup \left\{\left|\bar{L}_{1}(\varphi)(z)\right|: z \in \Pi_{2 \varepsilon}\right\}\|f\|
$$

So if we let $\varepsilon$ go to zero and use the fact that $\left\|\bar{\partial}^{*} N\right\| \leq \sqrt{e} \tau_{\Omega}$, we get

$$
\left\|H_{\varphi}\right\|_{e} \leq \sqrt{e} \tau_{\Omega} \sup \left\{\left|\bar{L}_{1}(\varphi)(z)\right|: z \in \Pi\right\} \text {. }
$$

On the other hand, for $p \in \Pi$ there exist $p_{j} \in \Pi, \xi_{j} \in \mathbb{D}$, and $F_{j} \in \Gamma_{b \Omega}$ such that $F_{j}\left(\xi_{j}\right)=p_{j}$ and $\lim p_{j}=p$. We note that if $p$ is not on the boundary of a disk then we can choose $p_{j}=p$ for all $j$.

Let $F_{j}(\xi)=\left(F_{j 1}(\xi), F_{j 2}(\xi)\right)$ for $\xi \in \mathbb{D}$. Since $\Omega$ is convex in $\mathbb{C}^{2}$ and we assume that $p_{j}$ is in a horizontal disk, $F_{j 1}$ is linear and $F_{j 2}$ is constant. The chain rule and the fact that $L_{1}$ is the complex tangential derivative imply that

$$
\left(\varphi \circ F_{j}\right)_{\bar{\xi}}\left(\xi_{j}\right)=\varphi_{\bar{z}}\left(p_{j}\right) \overline{F_{j 1}\left(\xi_{j}\right)}=\bar{L}_{1}(\varphi)\left(p_{j}\right) \overline{F_{j 1}^{\prime}\left(\xi_{j}\right)}=\bar{L}_{1}(\varphi)\left(p_{j}\right) \overline{F_{j 1}^{\prime}(0)}
$$

Hence

$$
\left|\bar{L}_{1}(\varphi)\left(p_{j}\right)\right|=\frac{\left|\left(\varphi \circ F_{j}\right)_{\bar{\xi}}\left(\xi_{j}\right)\right|}{\left|\overline{F_{j}^{\prime}(0)}\right|}
$$


Then, if we take supremum over $j$ we get

$$
\left|\bar{L}_{1}(\varphi)(p)\right| \leq \sup _{j} \sup _{\xi \in \mathbb{D}}\left\{\frac{\left|\left(\varphi \circ F_{j}\right)_{\bar{\zeta}}(\xi)\right|}{\left|F_{j}^{\prime}(0)\right|}\right\} .
$$

Hence, we have

$$
\left\|H_{\varphi}\right\|_{e} \leq \sup _{F \in \Gamma_{b \Omega}}\left\{\frac{\sqrt{e} \tau_{\Omega}}{\left|F^{\prime}(0)\right|} \sup _{\xi \in \mathbb{D}}\left\{\left|(\varphi \circ F)_{\bar{\xi}}(\xi)\right|\right\}\right\} .
$$

This completes the proof of Theorem 1 .

Proof of Theorem 2] The proof of Theorem 2 is very similar to the first part of the proof of Theorem 1. So instead of running through the whole argument again we will point out where they differ and the modifications needed for this proof. Without of loss of generality we may assume that there exists $z_{0} \in \mathbb{D}, p \in b \mathbb{D}$ such that $\varphi_{\bar{z}}\left(z_{0}, p\right) \neq 0$. In this case wedge $W$ is replaced by the disk $\mathbb{D}$ in $w$. Let us choose a sequence $\left\{p_{j}\right\} \subset \mathbb{D}$ such that $\lim _{j \rightarrow \infty} p_{j}=p$. Let us define $f_{j}(w)=k_{p_{j}}(w)$ where $k_{p_{j}}$ is the normalized Bergman kernel of $\mathbb{D}$ centered at $p_{j}$. Then instead of (1) we have

$$
\left\|f_{j}\right\|_{\mathbb{D}}=1 \text { and }\left\|f_{j}\right\|=\sqrt{\pi}
$$

The decomposition of $\varphi$ is unnecessary in case of the bidisk. Or simply we decompose $\varphi=\varphi_{1}+\varphi_{2}$ where $\varphi_{1}=\varphi$ and $\varphi_{2}=0$. We choose $D \subset \mathbb{D} \times\{p\}$ such that $\left(z_{0}, p\right) \in D$ and $\varphi_{\bar{z}}$ does not vanish on $D$. In a similar fashion as in the proof of Theorem 1 we get the following inequality.

$$
\left\|f_{j}\right\|_{\mathbb{D}} \frac{\int_{D} \chi(z) d V(z)}{\left\|\gamma_{\bar{z}}\right\|_{D}} \leq\left\|H_{\varphi} f_{j}\right\|_{D \times \mathbb{D}} \leq\left\|H_{\varphi} f_{j}\right\|
$$

Then

$$
\left\|H_{\varphi} f_{j}\right\| \geq \frac{V(D)}{\sqrt{2 \pi}}\left(\inf _{D}\left|\varphi_{\bar{z}}\right|\right) .
$$

We can estimate the essential norm as in (6)

$$
\left\|H_{\varphi}\right\|_{e} \geq \limsup _{j \rightarrow \infty} \frac{\left\|H_{\varphi} f_{j}\right\|}{\sqrt{\pi}}-\varepsilon
$$

for an arbitrary $\varepsilon>0$. Furthermore, we choose $r>0$ so that $F(\xi)=\left(r\left(\xi-z_{0}\right), p\right)$ and $D=F(\mathbb{D})$. Then

$$
V(D) \inf _{\xi \in D}\left\{\left|\varphi_{\bar{z}}(\xi)\right|\right\}=\pi\left|F^{\prime}(0)\right| \inf _{\xi \in \mathbb{D}}\left\{\left|(\varphi \circ F)_{\bar{\xi}}(\xi)\right|\right\} .
$$


Hence

$$
\left\|H_{\varphi}\right\|_{e} \geq \limsup _{j \rightarrow \infty} \frac{\left\|H_{\varphi_{1}} f_{j}\right\|}{\sqrt{\pi}}-\varepsilon \geq \frac{\left|F^{\prime}(0)\right|}{\sqrt{2}} \inf _{\xi \in \mathbb{D}}\left\{\left|(\varphi \circ F)_{\bar{\zeta}}(\xi)\right|\right\}-\varepsilon .
$$

Now we take supremum over $F$ and let $\varepsilon$ go to zero

$$
\left\|H_{\varphi}\right\|_{e} \geq \sup _{F \in \Gamma_{b \mathbb{D}}}\left\{\frac{\left|F^{\prime}(0)\right|}{\sqrt{2}} \inf _{\xi \in \mathbb{D}}\left\{\left|(\varphi \circ F)_{\bar{\zeta}}(\xi)\right|\right\}\right\} .
$$

This completes the proof of Theorem 2 .

\section{REFERENCES}

[AAK71] V. M. Adamjan, D. Z. Arov, and M. G. Kreĭn, Analytic properties of the Schmidt pairs of a Hankel operator and the generalized Schur-Takagi problem, Mat. Sb. (N.S.) 86(128) (1971), 34-75.

[Ass00] S. Asserda, The essential norm of Hankel operator on the Bergman spaces of strongly pseudoconvex domains, Integral Equations Operator Theory 36 (2000), no. 4, 379-395.

[CS01] So-Chin Chen and Mei-Chi Shaw, Partial differential equations in several complex variables, AMS/IP Studies in Advanced Mathematics, vol. 19, American Mathematical Society, Providence, RI; International Press, Boston, MA, 2001.

[ČŞ09] Željko Čučković and Sönmez Şahutoğlu, Compactness of Hankel operators and analytic discs in the boundary of pseudoconvex domains, J. Funct. Anal. 256 (2009), no. 11, 3730-3742.

[FS98] Siqi Fu and Emil J. Straube, Compactness of the $\bar{\partial}$-Neumann problem on convex domains, J. Funct. Anal. 159 (1998), no. 2, 629-641.

[JP93] Marek Jarnicki and Peter Pflug, Invariant distances and metrics in complex analysis, de Gruyter Expositions in Mathematics, vol. 9, Walter de Gruyter \& Co., Berlin, 1993.

[LR93] Peng Lin and Richard Rochberg, The essential norm of Hankel operator on the Bergman space, Integral Equations Operator Theory 17 (1993), no. 3, 361-372.

[Str10] Emil J. Straube, Lectures on the $\mathcal{L}^{2}$-Sobolev theory of the $\bar{\partial}$-Neumann problem, ESI Lectures in Mathematics and Physics, vol. 7, European Mathematical Society (EMS), Zürich, 2010.

E-mail address: Zeljko.Cuckovic@utoledo.edu, Sonmez.Sahutoglu@utoledo.edu

University of Toledo, Department of MATHEMATics \& StATistics, TOledo, OH 43606, USA 